\title{
Relationship Between Electron Work Function And Crystal Lattice Constant
}

\author{
L. Korolevych, A. Borisov, H. Shevliakova \\ Department of Microelectronics \\ National Technical University of Ukraine "Igor Sikorsky Kyiv Polytechnic Institute" \\ Kyiv, Ukraine
}

\begin{abstract}
The paper presents a model of one-dimensional finite crystal. The calculation of the field of forces acting on an electron in a crystal was done. The relationship between the electron work function and the lattice constant was obtained. Show matching between theoretical values of work function and practical data.
\end{abstract}

Keywords - crystallophysics; MISFET-electronics; charge of surfase-states; crystal lattice; lattice constant; electron work function.

\section{INTRODUCTION}

As known, theory MIS electronics (and, in particular, theory MOS electronics) has some initial physical quantities which values are empirical or semi-empirical. These quantities included a electron work function $W_{e}$ [1] and a charge of surface states $Q_{s s}$ [2]. Moreover, these quantities regardless of their different physical nature are similar between themselves. Since the electron work function can be identical to the work to be done to overcome the electrical forces acting at interface "material-vacuum". A charge of surface states which presented as localized electronic states at the interface of two substances, or, in the simplest case, at the interface of "material-vacuum." So these physical quantities can be described in terms of the field of forces acting on the electron near interface "materialvacuum". In general, this is a very difficult problem. So consider the case of the interface "crystal-vacuum".

The classical theory of crystalline lattice based on the consideration of crystalline substances in the form of homogeneous and continuous (infinity) substances. So classical theory can't be used to describe the finite crystal due to problems of describing physical and mathematical properties at interface "crystal-vacuum". That's can be resolved with using intersite aspect of crystalline lattice [3], [4], [5], [6].

\section{BASIS FOR ANALYSIS OF FORCE FIELD AT THE INTERFACE "CRYSTAL-VACUUM"}

So, we will be used intersite aspect crystal lattice for further calculations due to its advantages in comparison with classical theory. We also using one-dimensional approach for simplifying that calculations. By other words, we considering crystal in form of one-dimensional periodic series of formula complexes which are suspended in a vacuum at a temperature of absolute zero. That's allowing us to will ignore influences causes by temperature, time and macroscopic parameters of matter (like dielectric constant). Thus, we assume that crystal cell not has complex form, or, by other words, crystal cell have primitive form.
From the standpoint of crystal chemistry, crystal substance can be presented in one of two extreme models "ionic" or "covalent". So "ionic" crystal consist from even numbers of ions, i.e. in primitive cell - two ions, one of them is positive and second one is negative. The "covalent" crystal consist from regular spaced positive ions placed in electron liquid. It should be noted that the "ionic" model is suitable for describing ionic dielectric ( $\mathrm{LiF}, \mathrm{NaCl}$, etc.) and "covalent" model for semiconductors ( $\mathrm{Si}, \mathrm{Ge}$, etc.) or for metals $(\mathrm{Li}, \mathrm{Na}$, etc.). Because in theory of crystal lattice ions are often treated as point charges, we will do the same.

Based on above mentioned restrictions, internal crystal electric forces are determined by the laws of electrostatics only, such as Coulomb's law and the superposition principle. For reducing the impact of test charge to the force field, we assume that its mass is much smaller mass particles that form the crystal lattice.

As known, by the law of Coulomb, force acting on a test charge $q_{2}$ from the point charge $q_{1}$ defined as [7]:

$$
\vec{F}_{\mathrm{C}}=k_{\mathrm{C}} \frac{q_{1} q_{2}}{\left|\vec{r}_{12}\right|^{2}} \cdot \frac{\vec{r}_{12}}{\left|\vec{r}_{12}\right|},
$$

where $\vec{r}_{12}$ is radius vector directed from the charge $q_{1}$ to charge $q_{2}$ with a length equal to the distance between them; $k_{\mathrm{C}}-$ constant is equal to:

$$
k_{\mathrm{C}}=\left(4 \pi \varepsilon_{0}\right)^{-1},
$$

where $\varepsilon_{0}$ is vacuum permittivity.

If the system has more than two charges then result force acting on test charge is subject to the superposition principle [7]:

$$
\vec{F}_{k}=\sum_{\substack{n=1 \\ n \neq k}}^{m} \vec{F}_{n},
$$

where $\vec{F}_{n}$ is force for $n$th charge; $m$ is the total number of charges in the system. 
2017 IEEE First Ukraine Conference on Electrical and Computer Engineering (UKRCON)

\section{THE MAIN EQUATIONS FOR DETERMINATION OF FORCE DISTRIBUTION INSIDE/OUTSIDE CRYSTAL LATTICE}

Based on above assumptions, force acting on a test charge in case of "ionic" crystal will be determinated by forces from positive and negative immobile ions, in case of "covalent" crystal - by forces from positive ions and electron liquid (electron cloud). Consider the forces acting on a test charge in "covalent" case (Fig. 1). The force acting on a test charge from negative ions is same as force acting on a test charge from positive ions, but have opposite direction.

In order to analyze the forces acting on the test charge $q_{\mathrm{t}}$ from electron cloud, split it into parts $d X$ with charge $d q$ at coordinate $X$ (Fig. 1, b, d):

$$
d q=\eta d X
$$

Then force acting on a test charge $q_{\mathrm{t}}$ from charge $d q$ with accordance (3) and Coulomb's law (1) is:

$$
d \vec{F}=k_{\mathrm{C}} \frac{q^{2}}{a} \frac{d X}{|\vec{l}|^{2}} \cdot \frac{\vec{l}}{|\vec{l}|},
$$

where $\vec{l}$ is the radius vector directed as force acting on a test charge and equal to the distance between the charge $d q$ and the test charge $q_{\mathrm{t}}$ at coordinate $X_{\mathrm{t}}$ :

$$
|\vec{l}|=\left(X_{\mathrm{t}}-X\right)
$$

The result force acting on test charge from electron cloud side by superposition principle (3) will be equal to:

where $\eta=q / a$ is charge density of the electron cloud.

$$
\vec{F}_{\text {cloud }}=\int_{\text {cloud }} d \vec{F}
$$

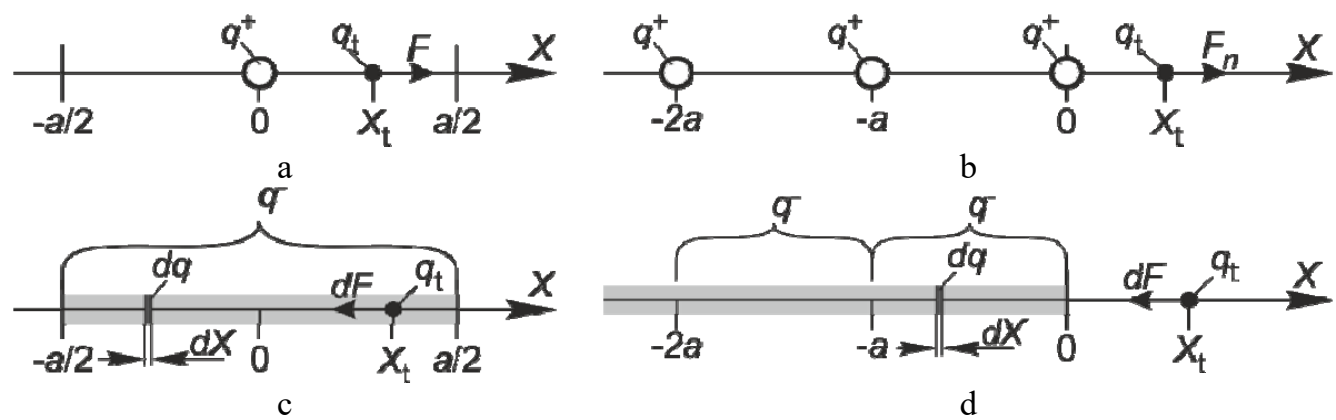

Fig. 1. Forces acting on test charge inside (a-b)/outside(c-d) crystal cell from ions (a, c) and electronic cloud (b, d)

The force acting on a test charge from positive ion inside crystal lattice (Fig. 1, a) in accordance to Coulomb's law (1):

$$
F_{\text {iic } 0}\left(X_{\mathrm{t}}\right)= \begin{cases}-k_{\mathrm{C}} \frac{q^{2}}{\left|X_{\mathrm{t}}\right|^{2}}, & X_{\mathrm{t}} \in[-\infty ; 0] \\ k_{\mathrm{C}} \frac{q^{2}}{\left|X_{\mathrm{t}}\right|^{2}}, & X_{\mathrm{t}} \in[0 ;+\infty]\end{cases}
$$

The force acting on a test charge from electronic cloud inside crystal lattice (Fig. 1, b) in accordance to a relations (47):

$$
F_{\text {eic } 0}\left(X_{\mathrm{t}}\right)=k_{\mathrm{C}} \frac{q^{2}}{a} \int_{-\frac{a}{2}}^{\frac{a}{2}} \begin{cases}\frac{d X}{\left(X_{\mathrm{t}}-X\right)^{2}}, & X \in\left[-\frac{a}{2} ; X_{\mathrm{t}}\right] \\ -\frac{d X}{\left(X_{\mathrm{t}}-X\right)^{2}}, & X \in\left[X_{\mathrm{t}} ; \frac{a}{2}\right] .\end{cases}
$$

or, equivalently:

$$
F_{\text {eic } 0}\left(X_{\mathrm{t}}\right)=k_{\mathrm{C}} \frac{q^{2}}{a} \frac{2 X_{\mathrm{t}}}{X_{\mathrm{t}}^{2}-(a / 2)^{2}}, \quad X_{\mathrm{t}} \in\left[-\frac{a}{2} ; \frac{a}{2}\right] .
$$

Similarly, obtain force acting on a test charge from semiinfinite electron cloud (Fig. 1, d):

$$
F_{\text {esi } 0}\left(X_{\mathrm{t}}\right)=-k_{\mathrm{C}} \frac{q^{2}}{a} \int_{-\infty}^{0}\left(X_{\mathrm{t}}-X\right)^{-2} d X, \quad X_{\mathrm{t}} \in[0 ;+\infty],
$$

or, equivalently:

$$
F_{\mathrm{esi} 0}\left(X_{\mathrm{t}}\right)=-k_{\mathrm{C}} \frac{q^{2}}{a} \cdot \frac{1}{X_{\mathrm{t}}} \quad X_{\mathrm{t}} \in[0 ;+\infty] .
$$

In accordance with superposition principle (3) and Coulomb's law (1), the force acting on a test charge from semi-infinite row of ions (Fig. 1, c) will be equal to:

$$
F_{\text {isi } 0}\left(X_{\mathrm{t}}\right)=k_{\mathrm{C}} q^{2} \sum_{n=0}^{+\infty}\left(X_{\mathrm{t}}+n \cdot a\right)^{-2}, \quad X_{\mathrm{t}} \in[0 ;+\infty] .
$$

By using [8]:

$$
\sum_{n=0}^{+\infty}(z+n)^{-2}=\Psi_{1}(z)
$$

where $\Psi_{1}(z)$ is a special function known as trigamma function, we can write relation (13) in next form:

$$
F_{\text {isi } 0}\left(X_{\mathrm{t}}\right)=\frac{k_{\mathrm{C}} q^{2}}{a^{2}} \Psi_{1}\left(\frac{X_{\mathrm{t}}}{a}\right), \quad X_{\mathrm{t}} \in[0 ;+\infty] .
$$


2017 IEEE First Ukraine Conference on Electrical and Computer Engineering (UKRCON)

For greater clarity, we will be made all further calculations in terms of relative length units:

$$
x=\frac{X_{t}}{a},
$$

with accordance to such scale factor:

$$
K_{F}=\frac{k_{\mathrm{C}} q^{2}}{a^{2}} .
$$

By using (16) and (17), relations (8), (10), (15) and (12) can be written in next forms:

$$
\begin{gathered}
f_{\text {iic } 0}(x)=\left\{\begin{array}{cc}
-x^{-2}, & x \in[-\infty ; 0] \\
x^{-2}, & x \in[0 ;+\infty] ;
\end{array}\right. \\
f_{\text {eic } 0}(x)=\frac{2 x}{x^{2}-(1 / 2)^{2}}, \quad x \in\left[-\frac{1}{2} ; \frac{1}{2}\right] ; \\
f_{\text {isi } 0}(x)=\Psi_{1}(x), \quad x \in[0 ;+\infty] ; \\
f_{\text {esi } 0}(x)=-x^{-1}, \quad x \in[0 ;+\infty]
\end{gathered}
$$

\section{THE FORCE DISTRIBUTION IN THE DEPTH OF CRYSTAL}

Consider force acting on a test charge in the cell of "ion" crystal in according to $x \in[-1 / 2 ; 1 / 2]$. The only 2 cases of one-dimensional "ionic" crystal presents: positive ion is placed left or right from cell center. Since radius of positive and negative ions are the same, then these cases differs by force direction only. Moreover, advisable is considering only gap between these ions $(x \in[-1 / 4 ; 1 / 4])$.

In case of ion row ending by negative charge, we obtain (22). Similarly, in case of opposite ending, we obtain (23). In case of "covalent" cell, the forces acting on a test charge in the depths of the crystal $(x \in[-1 / 2 ; 1 / 2])$, we obtain (24)

\section{THE FORCE DISTRIBUTION AT INTERFACE "CRYSTAL- VACUUM"}

The force acting on a test charge in a cell near the interface of "crystal-vacuum" different from the forces acting on the test charge in the depths of the crystal. This is due to the lack of charged particles in vacuum. It also causes features in the distribution of forces outside the crystal. Consider these features for "ion" and "covalent" crystal, provided that the interface "crystal-vacuum" matches with reference point $(x=0)$.

Since near the boundaries of "ionic" crystal can be both positive and negative ions, it is necessary to consider both cases. In the case of "ionic" crystal when ion row ends with a positive ion, force acting on a test charge from residuary half cell and outside the crystal will be equal (25) Similarly, in case when ion row ends with negative ion (26). Since near the boundaries of "covalent" crystal also consider two cases: when cell near interface "crystal-vacuum" is have or not have electron. In first case, we obtain (27). While in the second case, we obtain (28)

Comparing the expressions in the case of "ionic" crystal (22) and (23) with respectively (25) and (26), and in the case of "covalent" (24) to (27) seen that force distribution in the last cell is so close to force distribution in the depth of the crystal. Meanwhile, in "covalent' lattice case, force distribution (28) significantly differs from

$$
\begin{gathered}
f_{\mathrm{i} \mathrm{pn}}(x)=\Psi_{1}(x+1 / 4)-\Psi_{1}(x+3 / 4)+\Psi_{1}(1 / 4-x)-\Psi_{1}(3 / 4-x), x \in[-1 / 4 ; 1 / 4] ; \\
f_{\mathrm{inp}}(x)=\Psi_{1}(x+3 / 4)-\Psi_{1}(x+1 / 4)+\Psi_{1}(3 / 4-x)-\Psi_{1}(1 / 4-x), x \in[-1 / 4 ; 1 / 4] ; \\
f_{\mathrm{ic}}(x)= \begin{cases}-\mathrm{x}^{-2}+\Psi_{1}(x+1)-\Psi_{1}(-x+1), & x \in[-1 / 2 ; 0], \\
x^{-2}+\Psi_{1}(x+1)-\Psi_{1}(-x+1), & x \in[0 ; 1 / 2] ;\end{cases} \\
f_{\mathrm{i} \mathrm{ifp}}(x)= \begin{cases}\Psi_{1}(x+5 / 4)-\Psi_{1}(x+3 / 4)-(x+1 / 4)^{-2}, & x \in[-3 / 4 ;-1 / 4], \\
\Psi_{1}(x+5 / 4)-\Psi_{1}(x+3 / 4)+(x+1 / 4)^{-2}, & x \in[-1 / 4 ;+\infty] ;\end{cases} \\
f_{\mathrm{i} \mathrm{ifn}}(x)= \begin{cases}\Psi_{1}(x+3 / 4)-\Psi_{1}(x+5 / 4)+(x+1 / 4)^{-2}, & x \in[-3 / 4 ;-1 / 4], \\
\Psi_{1}(x+3 / 4)-\Psi_{1}(x+5 / 4)-(x+1 / 4)^{-2}, & x \in[-1 / 4 ;+\infty] .\end{cases} \\
f_{\mathrm{c} \mathrm{if} 1}(x)= \begin{cases}\Psi_{1}(x+3 / 2)-(x+1 / 2)^{-2}+x^{-1}, & x \in[-1 ;-1 / 2] ; \\
\Psi_{1}(x+3 / 2)+(x+1 / 2)^{-2}+x^{-1}, & x \in[-1 / 2 ; 0] ; \\
\Psi_{1}(x+3 / 2)+(x+1 / 2)^{-2}-x^{-1}, & x \in[0 ;+\infty] .\end{cases} \\
f_{\mathrm{cif} 2}(x)= \begin{cases}\Psi_{1}(x+3 / 2)-(x+1 / 2)^{-2}-(x+1)^{-1}, & x \in[-1 ;-1 / 2] ; \\
\Psi_{1}(x+3 / 2)+(x+1 / 2)^{-2}-(x+1)^{-1}, & x \in[-1 / 2 ;+\infty] .\end{cases}
\end{gathered}
$$




\section{THE CALCULATION OF WORK FUNCTION BASED ON THE ONE-DIMENSIONAL MODEL OF CRYSTAL}

Note that the available empirical values for work function lies in very wide range (upto $\pm 90 \%$ ), even for the same material [1], [9], which can be explained by influence uncontrolled surface conditions of investigated materials, methods of measuring and so on. Existing theoretical models to calculate the electron work function is not quantitative, complex and particular (usually they focus on a number of chemical elements, such as the of alkaline earth metals), but in terms of numerical values $W_{e}$ they tend to give much more or significantly less values than empirical ones [1]. Due to above we can't use this theoretic models in practice.

As known, work of force field on moving electric charge $q_{\mathrm{t}}$ from point with coordinate $X_{1}$ to point with coordinate $X_{2}$ is equal to difference between potential energy at these points:

$$
W_{F}=\Pi_{2}-\Pi_{1} \text {. }
$$

It should be noted that the expression (29) in case $\Pi_{1} \leq \Pi_{2}$, characterizes the work of force field on charge (positive projection of force field on move direction of test charge $q_{\mathrm{t}}$ - repulsive force), but in case $\Pi_{1}>\Pi_{2}$, characterizes the work of test charge against force field (negative projection of force field on move direction of test charge $q_{\mathrm{t}}$ - forces of attraction):

$$
W_{q}=-W_{F} .
$$

Obviously, the work (30) in conditions when negative test charge moved from the surface of material $\left(X_{1}=0\right)$ to infinity $\left(X_{2}=+\infty\right)$ is greater than zero, it coincides with the electron work function of the material [9].

On the other hand, $W_{F}$ is defined also as:

$$
W_{F}=\int_{X_{1}}^{X_{2}} F\left(X_{t}\right) d X_{t} .
$$

From expressions (30-31) and with condition $\left.F\left(X_{\mathrm{t}}\right)\right|_{X_{\mathrm{t}} \geq 0} \geq 0$ we obtain finally:

$$
W=-\int_{0}^{+\infty} F\left(X_{t}\right) d X_{t},
$$

Obviously, for calculating the electron work function from last cell of one-dimensional crystal we needs substitution in formula (32) expression for force field acting on negative test charge outside the crystal instead of general $F\left(X_{t}\right)$.

According to above described method of using relative units, we must introduce a scale factor:

$$
K_{W}=\frac{k_{\mathrm{C}} q^{2}}{a},
$$

then, in relative units and by the fact that we calculated forces for positive test charge, we obtain the following expression for the electron work function:

$$
A=\int_{0}^{+\infty} f(x) d x .
$$

Thus, in case while "ionic" crystal ends with negative ion we obtain according to expression (26):

$$
A_{\mathrm{in}}=\int_{0}^{+\infty} f_{\mathrm{i} \text { ifn }}(x) d x=-\pi,
$$

and in opposite case with according to (25) we obtain:

$$
A_{i p}=\pi \text {. }
$$

In case of negative ion on the surface, the repulsion forces act to electron and he does not need to do work against force field for move to infinity $(A<0)$, and in case of positive ion on the surface the electron must perform work on attraction forces for overcome to infinity $(A>0)$. That work in relative units equal to $\pi$. So, finally, electron work function from "ion" crystal can be written as (in relative units):

$$
A_{1}=\pi
$$

and in accordance to scale factor (33), we obtain finally:

$$
W_{\mathrm{i}}=7,2479 \cdot 10^{-19} \frac{1}{a} \mathrm{~J}=4,5238 \frac{1}{a} \mathrm{eV},
$$

where value $a$ is substituted in nanometers.

For calculate the electron work function from "covalent" crystal, take into account that surface of covalent crystal must be electro-neutral (each surface cell of crystal must have one positive ion and one electron cloud). So in this case, by integrating (27) with $x \geq 0$ from zero to infinity, we obtain:

$$
A_{\mathrm{c} 0}=\int_{0}^{+\infty} f_{\mathrm{c} \text { if } 1}(x) d x=-\infty .
$$

Due to (39), electron work function is negative, so we can conclude that on the surface are repulsive force. So, if last cell have inside it one electron then that electron is cannot move from it. Therefore, we consider the electron work function from last cell in condition when electron go out from last cell. So in this case, by integrating (28), we obtain:

$$
A_{\mathrm{c} 1}=\int_{0}^{+\infty} f_{\mathrm{c} \text { if } 2}(x) d x=\gamma+2 \ln 2 .
$$

So, finally, electron work function from "covalent" crystal can be write as (in relative units):

$$
A_{c}=\gamma+2 \ln 2 \approx 1,963510026
$$

and in accordance to scale factor (33), we obtain finally: 


$$
W_{\mathrm{c}}=4,5300 \cdot 10^{-19} \frac{1}{a} \mathrm{~J}=2,8274 \frac{1}{a} \mathrm{eV}
$$

where value $a$ is substituted in nanometers.

\section{THEORY OF WORK FUNCTION VS. PRACTICE}

Starting analyze of existing values of the work function for simple and complex crystalline materials [1], and comparing them with proper theoretical model ("covalent" and "ionic" crystals). Given the difference between the theoretical model for "covalent" and "ionic" crystals will consider this materials separately.

Given the fact that the theoretical model work function inversely proportional to the lattice parameter of onedimensional crystal is advisable to analyze the work function depending on parameter that inverse to lattice constant. Work function depending on inverse one-dimensional lattice parameter for all known crystals are shown in Fig. 2 and Fig. 3, where solid line is plotted by the method of least squares with condition of passing through zero (because the electron work function from crystal with an infinite lattice parameter must be equal zero).

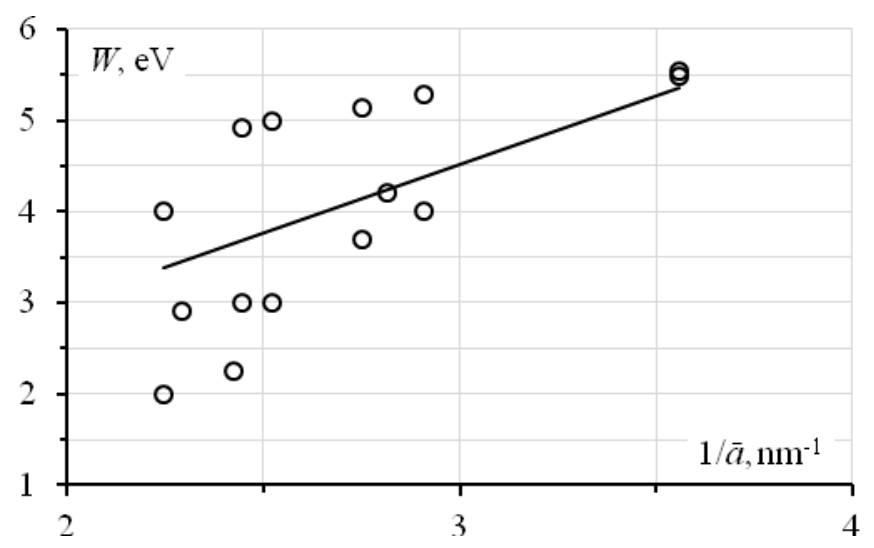

Fig. 2. The electron work function of "ionic" crystalline materials.

Algorithm of calculation lattice constant in onedimensional crystal model $(\bar{a})$ for each material is required a separate detailed consideration that cannot be considered within this paper.

From these, the least squares method, direct (see. Fig. 2 and Fig. 3) shows that the work function increases with decreasing lattice parameter of one-dimensional crystal, as needs and developed a theoretical model.

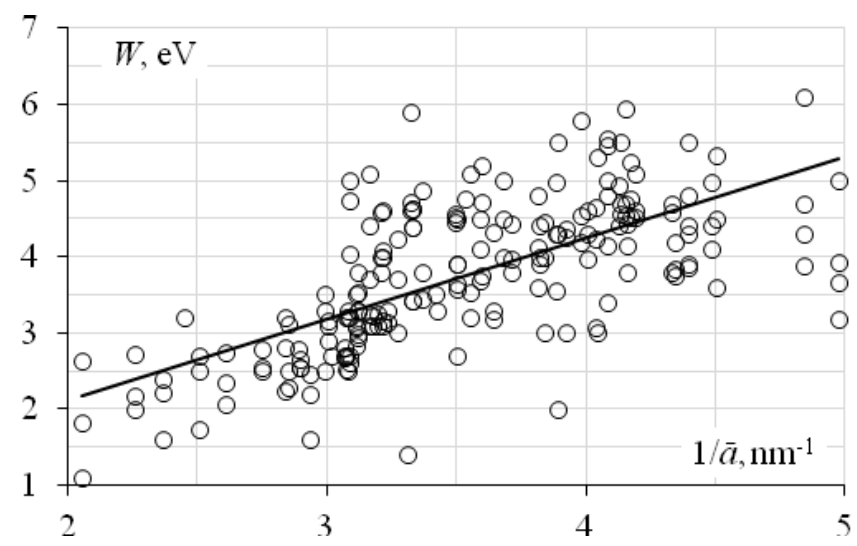

Fig. 3. The electron work function of "covalent" crystalline materials.

\section{CONCLUSIONS}

A model was developed one-dimensional finite crystal, based on inter-site aspect of crystal lattice. Two extreme cases of the crystal structure was considered: covalent and ionic.

Calculation of the forces acting on a electron in these crystals was done. Due to that theoretical relation for electron work function from the crystal was obtained. The last one is significally simplifies the solving for problem of choice proper material in design of heterojunction-based devices.

The correlation between theoretical values and practical data was shown.

Thus, this model of crystal can be the basis for solving some types of practical problems, e.g. for estimating the charge of surface states on the interface semiconductordielectric in MIS devices.

\section{REFERENCES}

[1] V.S. Fomenko, The emission properties of the materials. Handbook (in russian), Kyiv: Naukova dumka, 1981, p. 339.

[2] H.P. Peka, Physical phenomena on semiconductor surfaces (in russian), Kyiv: Vyshchia shkola. Golovne vydavnytstvo, 1984, p. 214.

[3] L. Korolevych, A. Borysov and M. Rodionov, "Intersite aspect aspect of crystal lattice. (in russian)," Electronics and Communications, 2011, vol. 4(63), pp. 32-38.

[4] L. Korolevych, A. Borysov and M. Rodionov, "Phenomenology of physical and mathematical description of concept of the crystal lattice (in russian)," Electronics and Communications, 2011, vol. 4(63), pp. 62-65.

[5] A. Borysov, L. Korolevych and H. Shevliakova., "Mathematical basics of intersite aspect of space crystal lattice. (in russian)," Electronics and Communications, 2016, vol. 5 (88), pp. 6-14.

[6] L. Korolevych, A. Borysov and H. Shevliakova., "Physical basics of intersite aspect of space crystal lattice. (in russian)," Electronics and Communications, in press.

[7] J. Rosen, Encyclopedia of Physics, Infobase Publishing, 2009, p. 365.

[8] E. Weisstein, CRC Concise Encyclopedia of Mathematics, Second ed., N.-Y.: Chapman \& Hall/CRC, 2002, p. 3252.

[9] L.N. Dobretsov and M.V. Homoiunova, Emission electronics (in russian), Moskow: Nauka, 1966, p. 564 